\title{
Optimal Control Modification Adaptive Law for Time-Scale Separated Systems
}

\author{
Nhan T. Nguyen
}

\begin{abstract}
Recently a new optimal control modification has been introduced that can achieve robust adaptation with a large adaptive gain without incurring high-frequency oscillations as with the standard model-reference adaptive control. This modification is based on an optimal control formulation to minimize the $\mathscr{L}_{2}$ norm of the tracking error. The optimal control modification adaptive law results in a stable adaptation in the presence of a large adaptive gain. This study examines the optimal control modification adaptive law in the context of a system with a time scale separation resulting from a fast plant with a slow actuator. A singular perturbation analysis is performed to derive a modification to the adaptive law by transforming the original system into a reduced-order system in slow time. A model matching conditions in the transformed time coordinate results in an increase in the actuator command that effectively compensate for the slow actuator dynamics. Simulations demonstrate effectiveness of the method.
\end{abstract}

\section{INTRODUCTION}

In the conventional MRAC framework, the tracking error is generally inversely proportional to the magnitude of the adaptive gain. However, a large adaptive gain can lead to high-frequency oscillations which can excite unmodeled dynamics that could adversely affect the stability of an MRAC law [1]. Various modifications were developed to increase robustness of MRAC by adding damping to the adaptive law to reduce high-frequency oscillations. Two well-known modifications in adaptive control are the $\sigma$-modification [2] and $\varepsilon_{1}$ - modification [3]. These modifications have been used extensively in adaptive control. Recently, a new modification has been introduced that is based on an optimal control formulation to minimize the $\mathscr{L}_{2}$-norm of the tracking error [4]. The optimality condition results in a damping term in the adaptive law proportional to the persistent excitation. The optimal control modification has been shown to be able to achieve fast adaptation with a large adaptive gain without compromising stability robustness while preserving tracking performance. This study extends the development of the optimal control modification adaptive law to the case when there exists a time-scale separation between a fast plant and a slow actuator which prevents the plant to follow a reference model even in the presence of adaptive control. A singular perturbation approach is used to separate the time scales of the plant and actuators and then modify the optimal control modification adaptive law to account for the slow actuator

Nhan T. Nguyen is with the Intelligent Systems Division at NASA Ames Research Center, Moffett Field, CA 94035 USA (e-mail: Nhan.T.Nguyen@nasa.gov) in the singularly perturbed system. The singular perturbation approach transforms the original system into a reduced-order system in slow time. A model matching condition is applied to the reduced-order system and the reference model in the transformed slow time coordinate that increases the actuator command to accommodate the slow actuator dynamics. The resulting control signal can then track the reference model better than if the actuator command is not modified.

\section{Singularly Perturbed Systems with Slow ACTUATORS}

Given a nonlinear plant as

$$
\dot{x}=A x+B\left[u+\Theta^{* \top} \Phi(x)+w(t)\right]
$$

where $x(t):[0, \infty) \rightarrow \mathbb{R}^{n}$ is a state vector, $u(t):[0, \infty) \rightarrow \mathbb{R}^{n}$ is a control vector, $A \in \mathbb{R}^{n \times n}$ and $B \in \mathbb{R}^{n \times n}$ are known matrices such that the pair $(A, B)$ is controllable and furthermore $A$ is Hurwitz and $B$ is invertible, $\Theta^{*} \in \mathbb{R}^{p \times n}$ is an unknown constant weight matrix, $\Phi(x): \mathbb{R}^{n} \rightarrow \mathbb{R}^{p}$ is a known bounded basis function and is at least piecewise smooth in $x$, and $w(t):[0, \infty) \rightarrow \mathbb{R}^{n}$ is a small unknown bounded disturbance with $\|w(t)\| \leq w_{0}$ and $\dot{w} \in \mathscr{L}_{\infty}$ for all $t$.

The controller $u(t)$ is subject to linear dynamics

$$
\dot{u}=\varepsilon \Lambda\left(u-u_{c}\right)
$$

where $u_{c}(t):[0, \infty) \rightarrow \mathbb{R}^{n}$ is an actuator command vector, $\Lambda \in \mathbb{R}^{n \times n}$ is a known Hurwitz matrix, and $\varepsilon$ is a positive constant and is assumed to be known by estimation.

The objective is to design the controller $u(t)$ that enables the plant to follow a reference model

$$
\dot{x}_{m}=A_{m} x_{m}+B_{m} r
$$

where $A_{m} \in \mathbb{R}^{n \times n}$ is Hurwitz and known, $B_{m} \in \mathbb{R}^{n \times m}$ is also known, and $r(t):[0, \infty) \rightarrow \mathbb{R}^{m} \in \mathscr{L}_{\infty}$ is a bounded command vector with $\dot{r} \in \mathscr{L}_{\infty}$.

Consider the case when $\varepsilon \ll 1$ is a small parameter and $\varepsilon\|\Lambda\| \ll\|A\|$. Then $x(t)$ is a fast state and $u(t)$ is a slow control. To decouple the fast and slow variables, the singular perturbation method is invoked using a slow time transformation

$$
\tau=\varepsilon t
$$

where $\tau$ is a slow time variable. 
Then, the plant and actuator models are transformed into a singularly perturbed system as

$$
\begin{gathered}
\varepsilon \frac{d x}{d \tau}=A x+B\left[u+\Theta^{* \top} \Phi(x)+w(t)\right] \\
\frac{d u}{d \tau}=\Lambda\left(u-u_{c}\right)
\end{gathered}
$$

The Tikhonov's theorem can be used to approximate the solution of the singularly perturbed system with the solution of a "reduced-order" system by setting $\varepsilon=0$ [5]. Then, $x(u, w, \varepsilon)$ is on a fast manifold. Thus, the reduced-order system is given by

$$
\begin{aligned}
B^{-1} A x_{0}+u_{0}+\Theta^{* \top} \Phi\left(x_{0}\right) & +w\left(\frac{\tau}{\varepsilon}\right) \\
& =u_{0}+w\left(\frac{\tau}{\varepsilon}\right)+f\left(x_{0}\right)=0 \\
\frac{d u_{0}}{d \tau}= & \Lambda\left(u_{0}-u_{c}\right)
\end{aligned}
$$

where $x_{0}$ and $u_{0}$ are the "outer" solution of the singularly perturbed system.

The "inner" or "boundary layer" solution for this system is obtained from

$$
\begin{gathered}
\dot{x}_{i}=A x_{i}+B\left[u_{i}+\Theta^{* \top} \Phi\left(x_{i}\right)+w(t)\right] \\
u_{i}-u_{c}=0
\end{gathered}
$$

The solution is then expressed as

$$
x(t)=x_{0}(t)+x_{i}(t)-x_{M A E}(t)
$$

where $x_{M A E}(t)$ is a correction term by a matched asymptotic expansion method applied to both the inner and outer solutions [6]. The outer solution is in fact the asymptotic solution of the original system as $t \rightarrow \infty$.

The algebraic solution of Eq. (7) can be expressed in general as

$$
x_{0}\left(u_{0}, w, \varepsilon\right)=g\left(u_{0}+w\left(\frac{\tau}{\varepsilon}\right)\right)=-f^{-1}\left(u_{0}+w\left(\frac{\tau}{\varepsilon}\right)\right)
$$

assuming $f^{-1}$ exists.

Differentiating Eq. (11) with respect to the slow time variable and then substituting the actuator model into the result yield

$\frac{d x_{0}}{d \tau}=\frac{\partial g}{\partial u_{0}} \Lambda\left[-B^{-1} A x_{0}-\Theta^{* \top} \Phi\left(x_{0}\right)-w\left(\frac{\tau}{\varepsilon}\right)-u_{c}\right]+\frac{\partial g}{\partial w} \frac{d w}{d \tau}$

From Eq. (7)

$$
\frac{\partial g}{\partial u_{0}}=\frac{\partial g}{\partial w}=-\left[B^{-1} A+\Theta^{* \top} \Phi^{\prime}\left(x_{0}\right)\right]^{-1}
$$

Consider asymptotic solution of the singularly perturbed system. Then, in slow time, the reference model is expressed as

$$
\frac{d x_{m}}{d \tau}=\frac{1}{\varepsilon}\left(A_{m} x_{m}+B_{m} r\right)
$$

Note that since $\partial g / \partial u_{0}$ contains the uncertainty, the control design is quite complicated. In order to simplified the solution, the uncertainty term is assumed to be small. That is

$$
\left\|\Theta^{* \top} \Phi^{\prime}(x)\right\| \ll\left\|B^{-1} A\right\|
$$

Then, using the matrix inversion lemma

$$
\begin{aligned}
& {\left[B^{-1} A+\Theta^{* \top} \Phi^{\prime}(x)\right]^{-1} }=A^{-1} B \\
&-A^{-1} B\left[\left(\Theta^{* \top} \Phi^{\prime}(x)\right)^{-1}+\left(B^{-1} A\right)^{-1}\right]^{-1} A^{-1} B \\
& \approx\left[I-A^{-1} B \Theta^{* \top} \Phi^{\prime}(x)\right] A^{-1} B
\end{aligned}
$$

The following choice for the actuator command is made

$$
u_{c}=K_{x} x+K_{r} r-u_{a d}
$$

where

$$
\begin{gathered}
K_{x}=\Lambda^{-1} B^{-1} A \frac{1}{\varepsilon} A_{m}-B^{-1} A \\
K_{r}=\Lambda^{-1} B^{-1} A \frac{1}{\varepsilon} B_{m}
\end{gathered}
$$

Using the result of the matrix inversion lemma, the closedloop singularly perturbed system now becomes

$$
\begin{aligned}
\frac{d x}{d \tau}= & {\left[I-A^{-1} B \Theta^{* \top} \Phi^{\prime}(x)\right] \frac{1}{\varepsilon}\left(A_{m} x+B_{m} r\right) } \\
& +\frac{\partial g}{\partial u} \Lambda\left[u_{a d}-\Theta^{* \top} \Phi(x)-w\left(\frac{\tau}{\varepsilon}\right)\right]+\frac{\partial g}{\partial w} \frac{d w}{d \tau}
\end{aligned}
$$

Then, the adaptive signal $u_{a d}$ can be designed to keep the following expression small by a judicious choice of a new basis function $\Phi_{1}(x, r): \mathbb{R}^{n} \times \mathbb{R}^{m} \rightarrow \mathbb{R}^{q}$ that spans the unknown constant parameter space $\Theta_{1}^{*} \in \mathbb{R}^{q \times n}$ such that

$$
\begin{gathered}
-A^{-1} B \Theta^{* \top} \frac{d \Phi(x)}{d x} \frac{1}{\varepsilon}\left(A_{m} x+B_{m} r\right) \\
+\frac{\partial g}{\partial u} \Lambda\left[u_{a d}-\Theta^{* \top} \Phi(x)-w\left(\frac{\tau}{\varepsilon}\right)\right]+\frac{\partial g}{\partial w} \frac{d w}{d \tau} \\
=-A^{-1} B \Lambda \tilde{\Theta}_{1}^{\top} \Phi_{1}(x, r)+\varphi(x, r)-\frac{\partial g}{\partial u} \Lambda w\left(\frac{\tau}{\varepsilon}\right)+\frac{\partial g}{\partial w} \frac{d w}{d \tau}
\end{gathered}
$$

where $\tilde{\Theta}_{1}=\Theta_{1}-\Theta_{1}^{*}$, and $\varphi(x, r)$ is an approximation error which is to be kept small by a suitable choice of basis functions.

Solving for $u_{a d}$ yields

$$
\begin{gathered}
u_{a d}=-\Lambda^{-1}\left[I+\Theta^{* \top} \Phi^{\prime}(x) A^{-1} B\right] \Theta^{* \top} \Phi^{\prime}(x) \frac{1}{\varepsilon}\left(A_{m} x+B_{m} r\right) \\
+\Theta^{* \top} \Phi(x)+\Lambda^{-1}\left(\frac{\partial g}{\partial u}\right)^{-1}\left[-A^{-1} B \Lambda \tilde{\Theta}_{1}^{\top} \Phi_{1}(x, r)+\varphi(x, r)\right]
\end{gathered}
$$

From the assumption in Eq. (15) and neglecting the term $\Delta=\Theta^{* \top} \Phi^{\prime}(x) A^{-1} B \Theta^{* \top} \Phi^{\prime}(x)$, then one possible choice for the new basis function is

$$
\Phi_{1}(x, r)=\left[\begin{array}{lll}
\Phi(x) & \Phi^{\prime}(x) x & \Phi^{\prime}(x) r
\end{array}\right]^{\top}
$$

Alternatively, the universal approximation theorem for neural networks can be used to approximate the uncertainty 
with a suitable choice of basis functions such as radial basis functions or sigmoidal basis functions [7].

The closed-loop plant model in slow time is expressed as

$$
\frac{d x}{d \tau}=\frac{1}{\varepsilon}\left(A_{m} x+B_{m} r\right)-\frac{1}{\varepsilon} B_{1} \tilde{\Theta}_{1}^{\top} \Phi_{1}(x)-\frac{1}{\varepsilon} B_{1} \delta\left(x, \frac{\tau}{\varepsilon}\right)
$$

where $\quad B_{1}=\varepsilon A^{-1} B \Lambda$ and $\delta\left(x, \frac{\tau}{\varepsilon}\right)=$ $-\Lambda^{-1} B^{-1} A\left\{\varphi\left(x, r\left(\frac{\tau}{\varepsilon}\right)\right)-\frac{\partial g}{\partial u} \Lambda w\left(\frac{\tau}{\varepsilon}\right)+\frac{\partial g}{\partial w} \frac{d w}{d \tau}+\mathscr{O}(\Delta x, \Delta r)\right\}$.

Since $A_{m}$ is Hurwitz and if $\tilde{\Theta}_{1}^{\top}$ is bounded, then the Tikhonov's theorem guarantees that the reduced solution with $\varepsilon>0$ converge to the solution of the original system with $\varepsilon=0$ as $\varepsilon \rightarrow 0$.

\section{Optimal Control Modification Adaptive LaW}

The tracking error equation in slow time is obtained as

$$
\frac{d e}{d \tau}=\frac{d x_{m}}{d \tau}-\frac{d x}{d \tau}=\frac{1}{\varepsilon} A_{m} e+\frac{1}{\varepsilon} B_{1}\left[\tilde{\Theta}_{1}^{\top} \Phi_{1}(x, t)+\delta\left(x, \frac{\tau}{\varepsilon}\right)\right]
$$

We are interested in seeking an update law for $\Theta$ that minimizes the following cost function in slow time

$$
J=\lim _{\tau_{f \rightarrow \infty}} \frac{1}{2 \varepsilon} \int_{0}^{\tau_{f}}(e-\Delta)^{\top} Q(e-\Delta) d \tau
$$

subject to Eq. (25) where $\Delta$ represents the unknown lower bound of the tracking error and $Q=Q^{\top}>0 \in \mathbb{R}^{n \times n}$.

This optimal control problem can be formulated by the Pontryagin's Maximum Principle. Defining a Hamiltonian

$$
\begin{aligned}
H\left(e, \tilde{\Theta}_{1}^{\top} \Phi_{1}\right)= & \frac{1}{2 \varepsilon}(e-\Delta)^{\top} Q(e-\Delta) \\
& +\frac{1}{\varepsilon} p^{\top}\left(A_{m} e+B_{1} \tilde{\Theta}_{1}^{\top} \Phi_{1}+B_{1} \delta\right)
\end{aligned}
$$

where $p(\tau):[0, \infty) \rightarrow \mathbb{R}^{n}$ is an adjoint variable, then the necessary condition is obtained as

$$
\frac{d p}{d \tau}=-\nabla H_{e}^{\top}=-\frac{1}{\varepsilon} Q(e-\Delta)-\frac{1}{\varepsilon} A_{m}^{\top} p
$$

with the transversality condition $p\left(\tau_{f}\right)=0$ since $e(0)$ is known.

The adaptive law which provides an optimal control solution can be formulated as a gradient update law as

$$
\frac{d \tilde{\Theta}_{1}}{d \tau}=\frac{d \Theta_{1}}{d \tau}=-\Gamma \nabla H_{\tilde{\Theta}_{1}}=-\frac{1}{\varepsilon} \Gamma \Phi_{1} p^{\top} B_{1}
$$

where $\Gamma=\Gamma^{\top}>0 \in \mathbb{R}^{q \times q}$ is an adaptive gain matrix.

An "approximate" solution of $p$ can be obtained using a "sweeping" method [8] by letting $p=P e+S \Theta_{1}^{\top} \Phi_{1}$, where $P=P^{\top}>0 \in \mathbb{R}^{n \times n}$ and $S \in \mathbb{R}^{n \times n}$. Substituting into the necessary condition yields

$$
\begin{aligned}
& \frac{d P}{d \tau} e+\frac{1}{\varepsilon} P\left(A_{m} e+B_{1} \tilde{\Theta}_{1}^{\top} \Phi_{1}+B_{1} \delta\right)+\frac{d S}{d \tau} \Theta_{1}^{\top} \Phi_{1} \\
+ & S \frac{d\left(\Theta_{1}^{\top} \Phi_{1}\right)}{d \tau}=-\frac{1}{\varepsilon} Q(e-\Delta)-\frac{1}{\varepsilon} A_{m}^{\top}\left(P e+S \Theta_{1}^{\top} \Phi_{1}\right)
\end{aligned}
$$

This results in the following equations obtained by a method of separation of variables

$$
\begin{gathered}
\frac{d P}{d \tau}+\frac{1}{\varepsilon}\left(P A_{m}+A_{m}^{\top} P\right)+\frac{1}{\varepsilon} Q=0 \\
\frac{d S}{d \tau}+\frac{1}{\varepsilon}\left(A_{m}^{\top} S+P B_{1}\right)=0 \\
-\frac{1}{\varepsilon} P B_{1}\left(\Theta_{1}^{* \top} \Phi-\delta\right)+S \frac{d\left(\Theta_{1}^{\top} \Phi\right)}{d \tau}-\frac{1}{\varepsilon} Q \Delta=0
\end{gathered}
$$

For an infinite time-horizon problem when $\tau_{f} \rightarrow \infty$, then $P(\tau) \rightarrow P(0)$ and $S(\tau) \rightarrow S(0)$ for all $t \in[0, \infty)$. So, both $P$ and $S$ tend to their constant solutions where

$$
\begin{gathered}
P A_{m}+A_{m}^{\top} P=-Q \\
S=-A_{m}^{-\top} P B_{1}
\end{gathered}
$$

Without loss of generality, a weighting constant $v>0 \in \mathbb{R}$ is introduced to allow for adjustments of the modification term in the adaptive law. Then, $v=1$ gives an optimal solution. Thus, the adjoint $p$ becomes

$$
p=P e-v A_{m}^{-\top} P B_{1} \Theta_{1}^{\top} \Phi_{1}
$$

Substituting Eq. (36) into the gradient-based adaptive law yields the adaptive law in slow time

$$
\frac{d \Theta_{1}}{d \tau}=-\frac{1}{\varepsilon} \Gamma\left(\Phi_{1} e^{\top} P B_{1}-v \Phi_{1} \Phi_{1}^{\top} \Theta_{1} B_{1}^{\top} P A_{m}^{-1} B_{1}\right)
$$

Converting to regular time by multiplying $\varepsilon$ through Eq. (37) results in the optimal control modification adaptive law

$$
\dot{\Theta}_{1}=-\Gamma\left(\Phi_{1} e^{\top} P B_{1}-v \Phi_{1} \Phi_{1}^{\top} \Theta_{1} B_{1}^{\top} P A_{m}^{-1} B_{1}\right)
$$

\section{A. Stability Proof}

Choose a Lyapunov candidate function

$$
V=e^{\top} P e+\operatorname{trace}\left(\tilde{\Theta}_{1}^{\top} \Gamma^{-1} \tilde{\Theta}_{1}\right)
$$

Evaluating $d V / d \tau$ in slow time yields

$$
\begin{gathered}
\frac{d V}{d \tau}=\frac{1}{\varepsilon} e^{\top}\left(P A_{m}+A_{m}^{\top} P\right) e+\frac{2}{\varepsilon} e^{\top} P B_{1}\left(\tilde{\Theta}_{1}^{\top} \Phi_{1}+\delta\right) \\
-\frac{2}{\varepsilon} \operatorname{trace}\left(\tilde{\Theta}_{1}^{\top} \Phi_{1} e^{\top} P B_{1}-v \tilde{\Theta}_{1}^{\top} \Phi_{1} \Phi_{1}^{\top} \Theta_{1} B_{1}^{\top} P A_{m}^{-1} B_{1}\right)
\end{gathered}
$$

By the trace property trace $\left(X^{\top} Y\right)=Y X^{\top}$, then

$$
\begin{aligned}
& \frac{d V}{d \tau}=-\frac{1}{\varepsilon} e^{\top} Q e+\frac{2}{\varepsilon} e^{\top} P B_{1} \tilde{\Theta}_{1}^{\top} \Phi_{1}+\frac{2}{\varepsilon} e^{\top} P B_{1} \delta-\frac{2}{\varepsilon} e^{\top} P B \tilde{\Theta}_{1}^{\top} \Phi_{1} \\
& +\frac{2}{\varepsilon} \nu \Phi_{1}^{\top} \tilde{\Theta}_{1} B_{1}^{\top} P A_{m}^{-1} B \tilde{\Theta}_{1}^{\top} \Phi_{1}+\frac{2}{\varepsilon} \nu \Phi_{1}^{\top} \Theta_{1}^{*} B_{1}^{\top} P A_{m}^{-1} B \tilde{\Theta}_{1}^{\top} \Phi_{1}
\end{aligned}
$$

$P A_{m}^{-1}$ can be decomposed into a symmetric part $M=\frac{1}{2}\left(P A_{m}^{-1}+A_{m}^{-\top} P\right)=-\frac{1}{2} A_{m}^{-\top} Q A_{m}^{-1}<0$ and an antisymmetric part $N=\frac{1}{2}\left(P A_{m}^{-1}-A_{m}^{-\top} P\right)$. Then, $P A_{m}^{-1}=M+$ 
$N$. By the property of a symmetric matrix, since $M<0$, therefore $P A_{m}^{-1}<0$. Thus

$$
\begin{aligned}
\frac{d V}{d \tau}= & -\frac{1}{\varepsilon} e^{\top} Q e+\frac{2}{\varepsilon} e^{\top} P B_{1} \delta \\
+\frac{2}{\varepsilon} \nu \Phi_{1}^{\top} \tilde{\Theta}_{1} B_{1}^{\top}( & \left.-\frac{1}{2} A_{m}^{-\top} Q A_{m}^{-1}+N\right) B_{1} \tilde{\Theta}_{1}^{\top} \Phi_{1} \\
& +\frac{2}{\varepsilon} \nu \Phi_{1}^{\top} \Theta_{1}^{*} B_{1}^{\top} P A_{m}^{-1} B_{1} \tilde{\Theta}_{1}^{\top} \Phi_{1}
\end{aligned}
$$

Using the property of an anti-symmetric matrix $y^{\top} N y=0$, $d V / d \tau$ is then bounded by

$$
\begin{aligned}
\frac{d V}{d \tau} \leq-\frac{1}{\varepsilon} \lambda_{\min }(Q)\|e\|\left[\|e\|-2\left\|P B_{1}\right\| \delta_{0}\right] & \\
& -\frac{1}{\varepsilon} v \lambda_{\min }\left(A_{m}^{-\top} Q A_{m}^{-1}\right)\left\|B_{1}\right\|^{2}\left\|\tilde{\Theta}_{1}\right\|\left\|\Phi_{1}\right\|^{2} \\
& {\left[\left\|\tilde{\Theta}_{1}\right\|-2\left\|P A_{m}^{-1}\right\| \Theta_{0}^{*}\right] }
\end{aligned}
$$

where $\delta_{0}=\sup _{x \in \mathscr{D}, \tau}\|\delta\|$ in some compact domain $\mathscr{D} \subset \mathbb{R}^{n}$, and $\Theta_{0}^{*}=\left\|\Theta_{1}^{*}\right\|$.

Let $B_{r}$ be a compact set where

$$
\begin{array}{r}
B_{r}=\left\{\left(e, \tilde{\Theta}_{1}\right) \in \mathbb{R}^{n} \times \mathbb{R}^{q \times n}:\|e\| \leq r=\frac{2\left\|P B_{1}\right\| \delta_{0}}{\lambda_{\min }(Q)},\right. \\
\left.\left\|\tilde{\Theta}_{1}\right\| \leq \kappa=\frac{2\left\|P A_{m}^{-1}\right\| \Theta_{0}^{*}}{\lambda_{\min }\left(A_{m}^{-\top} Q A_{m}^{-1}\right)}\right\}
\end{array}
$$

Then $d V / d \tau \leq 0$ in $B_{R}-B_{r}$ where $B_{r} \subset B_{R}=$ $\left\{e \in \mathbb{R}^{n}:\|e\| \leq R\right\} \subset \mathscr{D}$. Let $B_{\beta}$ be the smallest subset that encloses $B_{r}$, then there exists $\beta>0$ where

$$
\beta=\lambda_{\max }(P) r^{2}+\lambda_{\max }\left(\Gamma^{-1}\right) \kappa^{2}
$$

such that

$$
B_{r} \subset B_{\beta}=\left\{\left(e, \tilde{\Theta}_{1}\right) \in \mathbb{R}^{n} \times \mathbb{R}^{q \times n}: V \leq \beta\right\}
$$

Let $B_{\alpha}$ be the largest subset enclosed by $B_{R}$, then since $\|e\| \leq R$ in $B_{R}$, there exists $\alpha>0$ where

$$
\lambda_{\text {min }}(P)\|e\|^{2} \leq V \leq \lambda_{\text {min }}(P) R^{2}=\alpha
$$

such that

$$
B_{\alpha}=\left\{\left(e, \tilde{\Theta}_{1}\right) \in \mathbb{R}^{n} \times \mathbb{R}^{q \times n}: V \leq \alpha\right\} \subset B_{R}
$$

Then for a solution to be uniformly bounded, the set containment is as follows:

$$
B_{r} \subset B_{\beta} \subset B_{\alpha} \subset B_{R}
$$

This implies

$$
\beta<\alpha \Leftrightarrow \lambda_{\max }(P) r^{2}+\lambda_{\max }\left(\Gamma^{-1}\right) \kappa^{2}<\lambda_{\min }(P) R^{2}
$$

Therefore

$$
R>\sqrt{\frac{\lambda_{\max }(P) r^{2}+\lambda_{\max }\left(\Gamma^{-1}\right) \kappa^{2}}{\lambda_{\min }(P)}}=\rho
$$

where $\rho$ is the smallest value of $R$.
Then $\rho$ is the ultimate bound of $e(t)$ such that

$$
r \leq\|e\| \leq \rho \leq R
$$

Since $d V / d \tau \leq 0$ for all $\left(e, \tilde{\Theta}_{1}\right) \in B_{R}-B_{r}$, therefore $V$ is a decreasing function of time outside of $B_{r}$. Thus, if $\left(e(0), \tilde{\Theta}_{1}(0)\right) \in B_{\alpha}$, then according to Theorem 5.1 of Ref. [9], the solution will eventually enters $B_{\beta}$ after a finite time $t=T$ (independent of $(e(0), \tilde{\Theta}(0), \Delta \tilde{B}(0))$ and $\alpha$ ) and remain therein for all $t>T$. Therefore, $e(t)$ is uniformly ultimately bounded with an ultimate bound $\rho$.

Since $e(t)$ and $\tilde{\Theta}_{1}(t)$ are bounded, the unknown lower bound of the tracking error $\Delta$ at $t=t_{f} \rightarrow \infty$ is also bounded such that

$$
\|\Delta\| \leq \frac{\left\|P B_{1}\right\|\left(\beta+\delta_{0}+v \eta\left\|A_{m}^{-\top}\right\|\right)}{\lambda_{\min }(Q)}
$$

where $\left\|\Theta_{1}^{* \top} \Phi_{1}\right\| \leq \beta \in \mathbb{R}$ and $\left\|\frac{d\left(\Theta_{1}^{\top} \Phi_{1}\right)}{d t}\right\| \leq \eta \in \mathbb{R}$ for all $t$.

\section{B. Example}

Consider the following simple scalar system

$$
\dot{x}=a x+b u+\theta^{*} x+w(t)
$$

with actuator dynamics

$$
\dot{u}=\varepsilon \lambda\left(u-u_{c}\right)
$$

where $a<0, \lambda<0, \varepsilon>0,|\varepsilon \lambda|<|a|$, and $w(t)$ is a small disturbance signal.

The reference model is

$$
\dot{x}_{m}=a_{m} x_{m}+b_{m} r
$$

where $a_{m}<0$.

The actuator command is designed as

$$
u_{c}=\frac{a}{b}\left(\frac{a_{m}}{\varepsilon \lambda}-1\right) x+\frac{a b_{m}}{\varepsilon \lambda b} r-\Theta^{\top} \Phi(x, r)
$$

where $\Phi(x, r)=\left[\begin{array}{ll}x & r\end{array}\right]^{\top}$.

Note that if actuator dynamics are fast then the actuator command is

$$
u_{c}=\frac{a}{b}\left(\frac{a_{m}}{a}-1\right) x+\frac{b_{m}}{b} r-\theta x
$$

The optimal control modification update law for slow actuator system is

$$
\begin{aligned}
\dot{\Theta}=-\Gamma\left(\Phi e b_{1}\right. & \left.-v \Phi \Phi^{\top} \Theta_{1} \frac{b_{1}^{2}}{a_{m}}\right) \\
= & -\varepsilon \Gamma\left(\Phi e \frac{b \lambda}{a}-\varepsilon v \Phi \Phi^{\top} \Theta_{1} \frac{b^{2} \lambda^{2}}{a^{2} a_{m}}\right)
\end{aligned}
$$

where $b_{1}=\frac{\varepsilon \lambda b}{a}$, and for fast actuator system is

$$
\dot{\theta}=-\Gamma\left(x e b-v x^{2} \theta \frac{b^{2}}{a_{m}}\right)
$$

If $a$ and $\lambda$ are nominally in the same order of magnitude, then we note that for the slow actuator system, the effective 
adaptive gain is also reduced by $\varepsilon$ for a similar performance as that for the fast actuator.

For the numerical example, $a=-1, b=1, \theta^{*}=0.1$, $\lambda=-1, \varepsilon=0.1, a_{m}=-5, b_{m}=1, r(t)=\sin t, w(t)=$ $0.05 \sin 10 t, \Gamma=2850$, and $v=1$. The responses due to the standard MRAC adaptive law with and without the slow actuator compensation by the singular perturbation are plotted in Fig. 1. The adaptive gain $\Gamma$ was purposely selected high enough that the standard MRAC begins to exhibit instability due to slow actuator dynamics with the singular perturbation. The uncompensated response does not follow the reference model as expected.
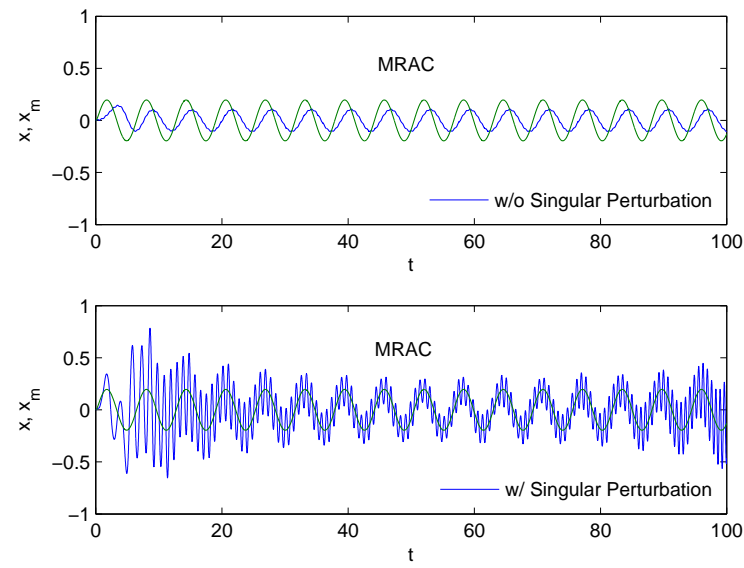

Fig. 1 - Response due to MRAC

The responses due to the optimal control modification adaptive law with and without the slow actuator compensation by the singular perturbation are plotted in Fig. 2 for the same adaptive gain $\Gamma$ and $v=1$. Without slow actuator compensation by the singular perturbation, the response cannot track the reference model. However, with slow actuator compensation by the singular perturbation, the optimal control modification produces a response that tracks the reference model very well.
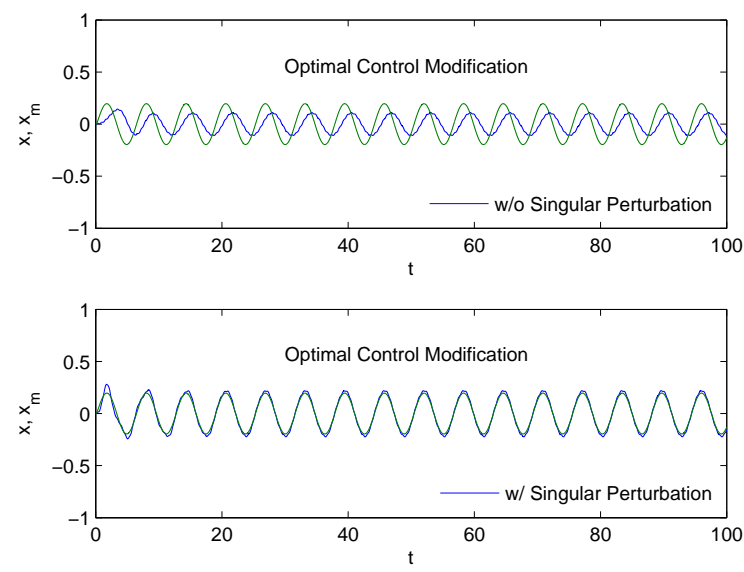

Fig. 2 - Response due to Optimal Control Modification

\section{Flight Control ExAmple}

Consider aircraft pitch attitude dynamics

$$
\begin{gathered}
{\left[\begin{array}{cc}
m V+\frac{C_{L_{\dot{\alpha}} \bar{q} S \bar{c}}}{2 V} & 0 \\
-\frac{C_{m_{\dot{\alpha}} \bar{q} S \bar{c}^{2}}}{2 V} & I_{y y}
\end{array}\right]\left[\begin{array}{c}
\dot{\alpha} \\
\dot{q}
\end{array}\right]=} \\
{\left[\begin{array}{cc}
-C_{L_{\alpha}} \bar{q} S & m V-\frac{C_{L_{q}} \bar{q} S \bar{c}}{2 V} \\
C_{m_{\alpha}} & \frac{C_{m_{q}} \bar{q} S \bar{c}^{2}}{2 V}
\end{array}\right]\left[\begin{array}{c}
\alpha \\
q
\end{array}\right]+\left[\begin{array}{c}
-C_{L_{\delta_{e}}} \\
C_{m_{\delta_{e}}}
\end{array}\right]\left(\delta_{e}+\Theta^{* \top} \Phi\right)}
\end{gathered}
$$

where $\Phi=\left[\begin{array}{ll}\alpha & q\end{array}\right]^{\top}$ and $\Theta^{* \top}=\left[\begin{array}{ll}0 & -0.1657\end{array}\right]$ is a parametric uncertainty that represents an $80 \%$ reduction in the pitch damping coefficient $C_{m_{q}}$.

A numerical model for a full-scale generic transport model (GTM) at Mach 0.8 and $30,000 \mathrm{ft}$ is given by

$$
\begin{gathered}
{\left[\begin{array}{c}
\dot{\alpha} \\
\dot{q}
\end{array}\right]=\underbrace{\left[\begin{array}{cc}
-0.7018 & 0.9761 \\
-2.6923 & -0.7322
\end{array}\right]}_{A}\left[\begin{array}{c}
\alpha \\
q
\end{array}\right]} \\
+\underbrace{\left[\begin{array}{l}
-0.0573 \\
-3.5352
\end{array}\right]}_{B}\left(\delta_{e}-0.1657 q\right)
\end{gathered}
$$

The elevator output is prescribed by degraded actuator dynamics

$$
\dot{u}=\varepsilon \lambda\left(u-u_{c}\right)
$$

where $\lambda=50 \mathrm{rad} / \mathrm{sec}$ and $\varepsilon=0.01$.

A desired reference model of the pitch attitude is given by

$$
\left[\begin{array}{c}
\dot{\theta}_{m} \\
\dot{q}_{m}
\end{array}\right]=\underbrace{\left[\begin{array}{cc}
0 & 1 \\
-\omega_{n}^{2} & -2 \zeta \omega_{n}
\end{array}\right]}_{A_{m}}\left[\begin{array}{c}
\theta_{m} \\
q_{m}
\end{array}\right]+\underbrace{\left[\begin{array}{c}
0 \\
\omega_{n}^{2}
\end{array}\right]}_{B_{m}} r
$$

where $\zeta=0.85$ and $\omega_{n}=1.5 \mathrm{rad} / \mathrm{sec}$ are chosen to give a desired handling characteristic.

Let $x=\left[\begin{array}{cc}\theta & q\end{array}\right]^{\top}$ and $q=C x$ where $C=\left[\begin{array}{ll}0 & 1\end{array}\right]$. A desired nominal controller is designed as $u^{*}=k_{\alpha}^{*} \alpha+$ $K_{x}^{*} x+k_{r}^{*} r$ where $k_{\alpha}^{*}=-\frac{a_{21}}{b_{2}}=-0.7616, K_{x}^{*}=\frac{C\left(A_{m}-a_{22} I\right)}{b_{2}}=$ $\left[\begin{array}{ll}-\frac{\omega_{n}^{2}}{b_{2}} & -\frac{2 \zeta \omega_{n}+a_{22}}{b_{2}}\end{array}\right]=\left[\begin{array}{ll}0.6365 & 0.5142\end{array}\right]$ and $k_{r}^{*}=$ $\frac{C B_{m}}{b_{2}}=\frac{\omega_{n}^{2}}{b_{2}}=-0.6365$. The closed-loop eigenvalues are -0.6582 and $-1.2750 \pm 0.7902 i$.

The actuator command without a compensation for the slow actuator dynamics is given by

$$
\begin{gathered}
u_{c}=k_{\alpha}^{*} \alpha+K_{x}^{*} x+k_{r}^{*} r-\Theta^{\top} \Phi \\
\dot{\Theta}=-\Gamma \Phi e P B
\end{gathered}
$$

where $e=x_{m}-x$.

Applying the singular perturbation method to compensate for the slow actuator dynamics, the reduced-order system is obtained as

$$
u=-\frac{a_{21}}{b_{2}} \alpha-\frac{a_{22}}{b_{2}} x-\theta^{*} q
$$

The compensated actuator command is then given by

$$
u_{c}=k_{\alpha}^{*} \alpha+K_{x} x+k_{r} r-\Theta^{\top} \Phi_{1}(\alpha, x, r)
$$


where $\Phi_{1}(\alpha, x, r)=\left[\begin{array}{ccc}\alpha & x & r\end{array}\right]^{\top}$ and

$$
\begin{gathered}
K_{x}=\frac{a_{22} C}{b_{2}}\left(\frac{A_{m}}{\varepsilon \lambda}-I\right) \\
k_{r}=\frac{a_{22} C B_{m}}{b_{2} \varepsilon \lambda} \\
\dot{\Theta}_{1}=-\Gamma \Phi_{1}\left(e^{\top} P-v \Phi_{1}^{\top} \Theta_{1} B_{1}^{\top} P A_{m}^{-1}\right) B_{1}
\end{gathered}
$$

where $B_{1}=\left[\begin{array}{ll}0 & \frac{b_{2} \varepsilon \lambda}{a_{22}}\end{array}\right]^{\top}$.

If $Q=q I>0$, then $B_{1}^{\top} P A_{m}^{-1} B_{1}=-\frac{q b_{2}^{2} \varepsilon^{2} \lambda^{2}}{2 a_{22}^{2} \omega_{n}^{4}}$ and the adaptive law can also be written as

$$
\dot{\Theta}_{1}=-\Gamma\left(\Phi_{1} e^{\top} P B_{1}+v \frac{q b_{2}^{2} \varepsilon^{2} \lambda^{2}}{2 a_{22}^{2} \omega_{n}^{4}} \Phi_{1} \Phi_{1}^{\top} \Theta_{1}\right)
$$

Figure 3 shows the pitch attitude response to a pitch doublet reference signal due to the standard MRAC. An adaptive gain of $\Gamma=100$ is used. Without compensation for the slow actuator dynamics, the pitch angle does not track the reference model. When the singular perturbation is used to compensate for the slow actuator dynamics, a high gain situation is encountered and the response produces a high frequency signal. This high gain is due to the factor of $1 / \varepsilon \lambda$ that appears in the control gains which effectively increases the actuator command to compensate for the slow actuator dynamics.
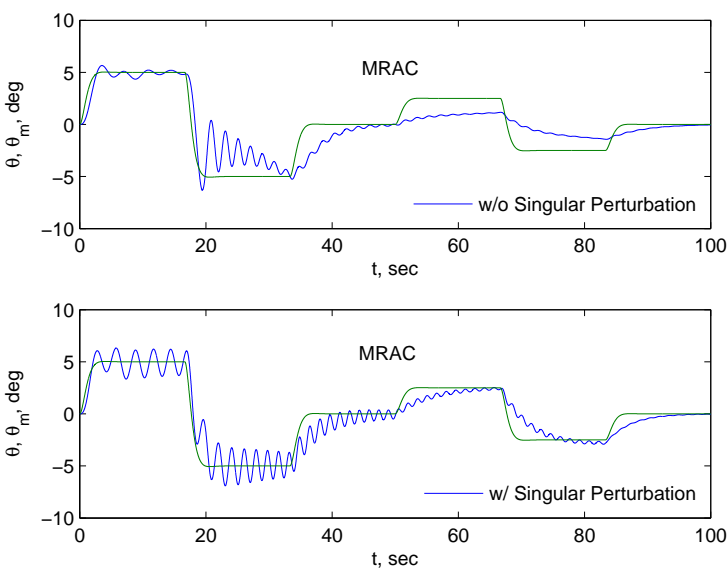

Fig. 3 - Pitch Attitude Response due to MRAC

Figure 4 shows the simulation results with the optimal control modification. The adaptive gain is kept the same and the tuning parameter $v=1$ is used. Without compensation, the pitch angle tracks the reference model reasonably well, although there is a delay in the response of about $1 \mathrm{sec}$. When the singular perturbation is used to compensate for the slow actuator dynamics, the delay is reduced, but in turn, the signal exhibits a higher frequency content.
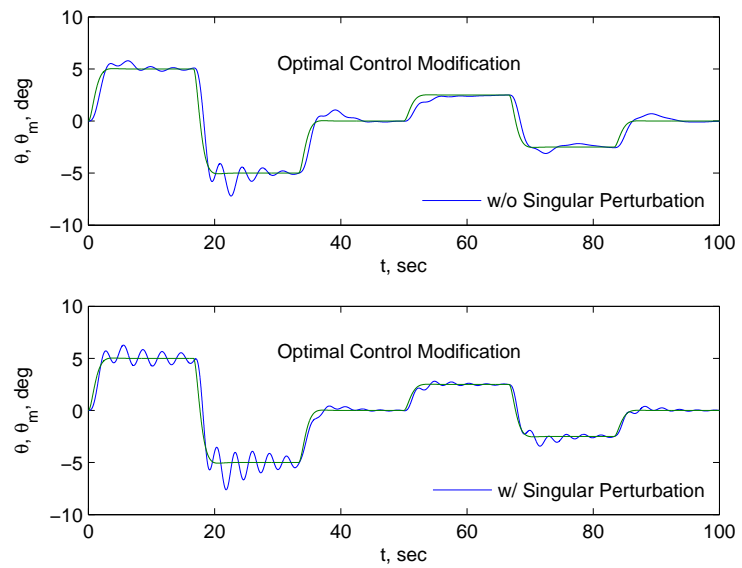

Fig. 4 - Pitch Attitude Responsse due to Optimal Control Modification

\section{CONCLUSIONS}

This paper presents a singular perturbation approach in connection with an optimal control modification adaptive law for a time-scale separated system with slow actuator dynamics. The singular perturbation approach transforms the system into a reduced-order system in a slow time coordinate. The actuator command is obtained by the model matching condition in the slow time coordinate. The resulting actuator signal in effect is increased by the ratio of the norm of the plant's transition matrix to the norm of the slow actuator's transition matrix. The optimal control modification adaptive is derived and analyzed for stability using the Lyapunov method. Under fast adaptation when the adaptive gain is large, the analysis shows that the tracking error remains bounded and stable. Simulation results demonstrate the effectiveness of the method.

\section{REFERENCES}

[1] Ioannu, P.A. and Sun, J. Robust Adaptive Control, Prentice-Hall, 1996. [2] Ioannou, P. and Kokotovic, P., "Instability Analysis and Improvement of Robustness of Adaptive Control," Automatica, Vol. 20, No. 5, 1984, pp. 583-594.

[3] Narendra, K.S. and Annaswamy, A.M., "A New Adaptive Law for Robust Adaptation Without Persistent Excitation", IEEE Transactions on Automatic Control, Vol. AC-32, No. 2, pp. 134-145, 1987.

[4] Nguyen, N., Krishnakumar, K., and Boskovic, J., "An Optimal Control Modification to Model-Reference Adaptive Control for Fast Adaptation", AIAA Guidance, Navigation, and Control Conference, AIAA 2008-7283, 2008.

[5] Kokotovic, P., Khalil, H., and O'reilly, J., Singular Perturbation Methods in Control: Analysis and Design, Society for Industrial and Applied Mathematics, 1987.

[6] Ardema, M., "Computational Singular Perturbation Method for Dynamical Systems", AIAA Journal of Guidance, Control, and Dynamics, Vol. 14, 661-663, 1981

[7] Cybenko, G., "Approximation by Superpositions of a Sigmoidal Function", Mathematics of Control Signals Systems, Vol. 2, pp. 303-314, 1989.

[8] Bryson, A.E. and Ho, Y.C., Applied Optimal Control: Optimization, Estimation, and Control, John Wiley \& Sons Inc., 1979.

[9] Khalil, H.K., Nonlinear Systems, Prentice-Hall, 2002. 